\title{
Erratum
}

\section{The Magnetisation of Large Atoms in Strong Magnetic Fields}

\section{S. Fournais}

Laboratoire de Mathématiques, Université Paris-Sud - Bât 425, 91405 Orsay Cedex, France.

E-mail: soeren.fournais@ math.u-psud.fr

Received: 22 April 2004 / Accepted: 23 April 2004

Erratum published online: 8 July 2004 - (C) Springer-Verlag 2004

Commun. Math. Phys. 216, 375-393 (2001)

The proof of [Fou01] contains an unjustified inequality. In Sect. 5, to get Eq. (15), it is tacitly used that

$$
\tilde{D}_{t}\left(\rho_{t, \lambda, \beta}-\rho_{\psi}, \rho_{t, \lambda, \beta}-\rho_{\psi}\right) \geq 0 .
$$

This is based on the idea that $\tilde{D}_{t}$ is positively definite, i.e. that

$$
\tilde{D}_{t}(f, f) \geq 0 \text {, for all } f \in \mathcal{D}\left(\tilde{D}_{t}\right) .
$$

Though $(0.1)$ is correct for positive $f$, it is not at all clear that it holds for functions with variable sign.

The remedy to this is to replace $\tilde{D}_{t}$ by another quadratic form, having the same Taylor expansion in $t$ to first order, and being clearly positive. This is accomplished by redefining $\tilde{D}_{t}$ as

$$
\tilde{D}_{t}(f, g)=\frac{1}{2} \iint_{\mathbb{R}^{3} \times \mathbb{R}^{3}} \frac{\overline{f(x)} g(y)}{\left|\phi_{t}(x)-\phi_{t}(y)\right|} d x d y,
$$

with $\phi_{t}$ being the function introduced in [Fou01, Appendix A]: $\phi_{t}(x+t \tilde{\mathbf{a}}(x))=x$. By a change of variables, and the positivity of the Coulomb kernel, this new $\tilde{D}_{t}$ is positively definite. Further details are given in [Fou03].

\section{References}

[Fou01] Fournais, S.: The magnetisation of large atoms in strong magnetic fields. Commun. Math. Phys. 216(2), 375-393 (2001)

[Fou03] Fournais, S.: Confinement to lowest Landau band and application to quantum current. Rev. Math. Phys. 15(10), 1219-1254 (2003) 